\title{
Supertranslations in higher dimensions revisited
}

\author{
Ankit Aggarwal* \\ The Institute of Mathematical Sciences, HBNI, CIT Campus, Chennai-600 113, India
}

(Received 16 November 2018; published 31 January 2019)

\begin{abstract}
In this paper, we revisit the question of identifying the soft graviton theorem in higher (even) dimensions with Ward identities associated with asymptotic symmetries. Building on the prior work of [Ann. Math. Sci. Appl. 2, 69 (2017)], we compute, from first principles, the (asymptotic) charges associated to supertranslation symmetry in higher even dimensions and show that (i) these charges are nontrivial and finite and (ii) the corresponding Ward identities are indeed the soft graviton theorems.
\end{abstract}

DOI: 10.1103/PhysRevD.99.026015

\section{INTRODUCTION}

Asymptotically flat spacetimes are those which approach flat spacetimes "far away" from the matter sources. A precise mathematical definition of asymptotic flatness involves specifying the boundary conditions-the rate at which the metric of the spacetime approaches the flat metric near its boundary. There is no unique prescription to specify these boundary conditions. Any particular choice is based on the following two physically motivated guiding principles (see the introduction of Ref. [1]). First, the conditions should be weak enough to allow for physically interesting solutions like black holes and gravitational radiation. Second, the conditions should be strong enough to ensure that the physically interesting notions like total mass or total radiated energy are finite and well defined.

The group of nontrivial transformations which leave the form of the specified boundary conditions invariant is known as the asymptotic symmetry group (ASG). The work of Bondi et al. [2] and Sachs [3,4] in the 1960s showed that the ASG of asymptotically flat spacetimes in four dimensions is the infinite-dimensional BondiMetzner-Sachs (BMS) group. It is a semidirect product of supertranslations (angle dependent translations along null infinity) and the Lorentz group. Curiously, however, the studies of asymptotically flat spacetimes in higher dimensions [1,5-7] have concluded that the supertranslations do not form a part of the ASG in $d>4$. This negative result is a consequence of using stringent boundary conditions that were guided by the physical considerations of

\footnotetext{
*aankita@imsc.res.in aankit1729@gmail.com

Published by the American Physical Society under the terms of the Creative Commons Attribution 4.0 International license. Further distribution of this work must maintain attribution to the author(s) and the published article's title, journal citation, and DOI. Funded by SCOAP ${ }^{3}$.
}

the kind mentioned above. A remarkably precise description of these considerations was provided in Ref. [1] for even $d>4$. First, it was shown that supertranslations are related to the memory effect and that in higher even dimensions memory effect is not present (to the order at which the tidal effects due gravitational radiation are seen). This was supplemented by the statement (see the conclusions of Ref. [1]) that if weaker boundary conditions are imposed to allow for supertranslations it would lead to an ill-defined charge (flux) for supertranslations (due to the divergence of symplectic current). Neither of these arguments holds in four dimensions. In fact, supertranslations are inevitable in $d=4$. Boundary conditions disallowing supertranslations automatically disallow all the generic radiative solutions (see e.g., Ref. [1]).

However, the absence of BMS (supertranslations) presents a puzzle. In a remarkable program initiated by Strominger et al. [8-17] (see Ref. [18] for a review), soft theorems have been understood to be the Ward identities associated with the asymptotic symmetries in gauge and gravitational theories. The Ward identities of BMS (and suitable extensions thereof) have been shown to be equivalent to the leading (subleading) soft graviton theorem in four dimensions [13-16]. In particular, in Ref. [13], a diagonal subgroup of the direct product of the BMS group at future $\left(\mathcal{I}^{+}\right)$and past null infinity $\left(\mathcal{I}^{-}\right)$was identified as a symmetry group of the quantum $\mathcal{S}$-matrix. The Ward identity for this symmetry group was then shown to be the leading/Weinberg soft graviton theorem [19] in four dimensions.

Furthermore, it is well understood that the soft graviton theorems exist in all dimensions [20-22]. Thus, a natural question to ask is whether or not the soft graviton theorems in higher dimensions are also statements about the symmetries of the quantum gravity $\mathcal{S}$-matrix. Motivated by this, the authors in Ref. [23] argued for the existence of supertranslations in even $d=2 m+2>4$. Starting from the leading soft graviton theorem, they wrote it as a Ward 
identity for the $\mathcal{S}$-matrix and read off a (conserved) charge. By proposing suitable commutation relations for radiative degrees of freedom, this charge was shown to generate supertranslations in even $d>4(m>1)$. Hence, results of Ref. [23] present a very compelling argument in favor of supertranslations in higher even dimensions. However, to unambiguously establish the existence of supertranslations in higher dimensions, one needs to show that (i) there exist suitable boundary conditions allowing them, (ii) the associated charge is finite and well defined, and (iii) the corresponding Ward identity is the leading soft graviton theorem. This was not established in Ref. [23], and the works of Refs. [1,5-7] seem to be at odds with this expectation. Relaxing the boundary conditions would allow supertranslations but would lead to an ill-defined charge as was pointed out in Ref. [1] (see the second paragraph above).

Thus, there is a tension between the results of classical general relativity and those of Ref. [23].

In this paper, we resolve this conundrum by proposing some additional boundary conditions near the boundaries of null infinity. As we shall see, these boundary conditions will lead to a finite, well-defined charge for supertranslations in higher even dimensions. We will also see that the boundary conditions that we propose can be thought of as analogs of Christodoulou-Klainermann (CK) constraints in four dimensions [9]. Doing this helps us get the right count of the number of independent leading soft graviton theorems (one). We will work in linearized gravity throughout the paper.

The outline of the paper is as follows. In Sec. II, we give the preliminaries needed for our work and explain the notation. Then, we do a detailed analysis of the sixdimensional case in Sec. III, where we compute the charges for supertranslations and give the generalized CK constraints in six dimensions. Our results are generalized to arbitrary higher even dimensions in Sec. IV. In Sec. V, we discuss the potential problems in going from the linear to the nonlinear regime. We conclude with a summary and future outlook in Sec. VI.

\section{PRELIMINARIES}

Our attention will be limited to arbitrary even dimensions because the notion of conformal null infinity breaks down in odd dimensions [24]. We will mostly use the notation of Ref. [23] and work in linearized gravity coupled to massless matter in $d=2 m+2$ dimensions. Linearization simplifies the analysis and is justified when there are no "hard" gravitons (gravitons with nonzero momentum) present in the external states. This will, in particular, mean that we will only keep the terms linear in metric fluctuations in the charge (the intermediate computations like those of symplectic potential would require us to keep terms quadratic in metric fluctuations since they contribute linearly to the charge). When there is no scope of confusion, the contraction of indices is denoted with a dot. We are interested in spacetimes that are asymptotically flat at both future and past null infinity. For concreteness, we focus on future null infinity; similar considerations apply to the past null infinity. Most of the content of this section (except Sec. II. D) can be found in Ref. [23] with more details. We work in units where $8 \pi G=1$, which is different from Ref. [23] where $32 \pi G=\kappa^{2}$.

\section{A. Metric and Bondi gauge}

We work in the Bondi coordinates $\left(u, r, z^{A}\right)$, where $u$ is the retarded time, $r$ is the radial coordinate, and $z^{A}$ are the coordinates on the sphere. The linearized $d$-metric is parametrized as

$$
d s^{2}=M d u^{2}-2 d u d r+g_{A B} d z^{A} d z^{B}-2 U_{A} d z^{A} d u,
$$

where $A, B=1, \ldots 2 m$ denote the sphere indices. The inverse metric is given by

$$
g^{\mu \nu}=\left(\begin{array}{ccc}
0 & -1 & 0 \\
-1 & -M & -U^{B} \\
0 & -U^{A} & g^{A B}
\end{array}\right) .
$$

We will assume $M, U_{A}$, and $g_{A B}$ admit an expansion near $\mathcal{I}^{+}$of the form:

$$
\begin{aligned}
M & =-1+\sum_{n=1}^{\infty} \frac{M^{(n)}(u, z)}{r^{n}}, \quad U_{A}=\sum_{n=0}^{\infty} \frac{U_{A}^{(n)}(u, z)}{r^{n}}, \\
g_{A B} & =r^{2} \gamma_{A B}+\sum_{n=-1}^{\infty} \frac{C_{A B}^{(n)}(u, z)}{r^{n}} .
\end{aligned}
$$

There is an additional determinant condition in the Bondi gauge,

$$
\operatorname{det}\left(g_{A B}\right)=r^{2 m} \operatorname{det}\left(\gamma_{A B}\right),
$$

where $\gamma_{A B}$ is the round metric on $S^{2 m}$. This condition, in linearized theory, implies that all the $C_{A B}^{(n)}$ are traceless,

$$
\gamma^{A B} C_{A B}^{(n)}=0 .
$$

\section{B. Boundary conditions and constraints from Einstein's equations}

Following Ref. [23], the boundary conditions for asymptotically flat spacetimes in arbitrary even dimensions are taken to be

$g_{\text {uи }}=-1+\mathcal{O}\left(r^{-1}\right), \quad g_{\text {ur }}=-1+\mathcal{O}\left(r^{-2}\right)$,

$g_{u A}=\mathcal{O}(1), \quad g_{A B}=r^{2} \gamma_{A B}+\mathcal{O}(r)$.

We also require

$$
\begin{aligned}
& R_{u и}=\mathcal{O}\left(r^{-2 m}\right), \quad R_{u r}=\mathcal{O}\left(r^{-2 m-1}\right), \\
& R_{u A}=\mathcal{O}\left(r^{-2 m}\right),
\end{aligned}
$$




$$
\begin{aligned}
R_{r r} & =\mathcal{O}\left(r^{-2 m-2}\right), \quad R_{r A}=\mathcal{O}\left(r^{-2 m-1}\right), \\
R_{A B} & =\mathcal{O}\left(r^{-2 m}\right) .
\end{aligned}
$$

Linearized Einstein's equations

$$
R_{m n}=\bar{\square} h_{m n}-2 \bar{\nabla}_{(m} \bar{\nabla} \cdot h_{n)}=T_{m n}
$$

determine metric components in terms of the free radiative as well as matter data. In Bondi coordinates, we get the following constraints.

The boundary condition on $R_{u r}$ reads

$$
\begin{aligned}
& -\frac{n(n+1-2 m)}{2} M^{(n)}+\frac{(n-1)}{2} D^{A} U_{A}^{(n-1)}=0, \\
& \quad 0 \leq n \leq 2 m-2 .
\end{aligned}
$$

The boundary condition on $R_{r A}$ reads

$$
\begin{aligned}
& \frac{(n+2)(n+1-2 m)}{2} U_{A}^{(n)}-\frac{(n+1)}{2} D^{B} C_{B A}^{(n-1)}=0, \\
& 0 \leq n \leq 2 m-2 .
\end{aligned}
$$

The $R_{A B}$ equations lead to

$$
\partial_{u} C_{A B}^{(-1)}=0 .
$$

This should be contrasted with the four dimensions, where (26) is not valid and $C_{A B}^{(-1)}$ is the free radiative data which, generically, can depend on $u$.

$R_{u и}$ equations give

$$
\begin{aligned}
& \frac{1}{2}\left[D^{2}-2(m-1)\right] M^{(2 m-2)}+\partial_{u} D^{A} U_{A}^{(2 m-2)}+m \partial_{u} M^{(2 m-1)} \\
& \quad+T_{u u}^{M(2 m)}=0 .
\end{aligned}
$$

where $T_{u u}^{M(2 m)}$ is the $\mathcal{O}\left(r^{-4}\right)$ component of matter stressenergy tensor which is assumed to have the same falloff behavior as the Ricci tensor. $C_{A B}^{(m-2)}$ is free radiative data (see Ref. [23]) as can be seen from saddle point approximation.

\section{Supertranslations in higher even dimensions}

In four dimensions, supertranslations form an infinitedimensional group (which is an Abelian subgroup of the BMS group). They are characterized by an arbitrary function on the sphere. In higher even dimensions, for the boundary conditions under consideration (see Sec. II B), there is an analogous infinite-dimensional group characterized by an arbitrary function of the sphere coordinates, $f(z)^{1}$ (see Ref. [23]). This will be referred to as supertranslations. Near $\mathcal{I}^{+}$, supertranslations are generated by the vector field

\footnotetext{
${ }^{1}$ It is also an Abelian subgroup of the analog of BMS in higher even dimensions.
}

$$
\xi=f \partial_{u}-\frac{1}{r} \gamma^{A B} D_{A} f \partial_{B}+\frac{1}{2 m} D^{2} f \partial_{r}+\cdots,
$$

where ... refer to subleading terms in $r$. It can be easily checked that the above vector field preserves the form of the metric near $\mathcal{I}^{+}$, making it a valid asymptotic symmetry.

The effect of the supertranslations is to shift $C_{A B}^{(-1)}$ according to

$$
\delta_{\xi} C_{A B}^{(-1)}=\frac{1}{m} D^{2} f \gamma_{A B}-\left(D_{A} D_{B}+D_{B} D_{A}\right) f .
$$

For the remaining $C_{A B}^{(n)} \mathrm{s}$,

$$
\delta_{\xi} C_{A B}^{(n \geq 0)}=\mathcal{O}(C) .
$$

Hence, in the linearized theory, ${ }^{2}$

$$
\delta_{\xi} C_{A B}^{(n \geq 0)}=0 .
$$

It is important to note that in four dimensions the free radiative data are $C_{A B}^{(-1)}$, which are affected by the supertranslations within the linearized regime. This is not so for higher dimensions where the free radiative data are $C_{A B}^{(m-2)}$ (as noted above), which remain unaffected by supertranslations in the linearized regime.

\section{Covariant phase space formalism}

We will be using covariant phase space formalism to compute the charge. This subsection enlists the steps involved in this computation (see Refs. [25-28] for a detailed discussion). The first step is to evaluate the symplectic potential

$$
\Theta_{t}(\delta):=\int_{\Sigma_{t}} d S_{a} \theta^{a}
$$

where

$$
\theta^{a}:=\frac{1}{2} \sqrt{g}\left(g^{b c} \delta \Gamma_{b c}^{a}-g^{a b} \delta \Gamma_{c b}^{c}\right)
$$

and $\Sigma_{t}$ is a $t=$ constant slice. Since $t:=r+u$, the component of interest will be $\theta^{t}=\theta^{r}+\theta^{u}$. The limit $t \rightarrow$ $\infty$ with $u$ constant takes us to $\mathcal{I}^{+}$, which is the surface on which the charge (flux) is computed. The variable $r$ will be understood as given by $r=t-u$ while performing the integration on the spatial slice.

Using symplectic potential, the standard covariant phase space symplectic form can be computed,

\footnotetext{
${ }^{2}$ Since we are working in the linearized theory, we have ignored the terms homogeneous in metric perturbations because these will lead to nonlinear terms in charge as will become clear in the following sections.
} 


$$
\Omega_{t, g}\left(\delta, \delta^{\prime}\right):=\int_{\Sigma_{t}} d S_{a} \omega_{g}^{a}\left(\delta, \delta^{\prime}\right)
$$

where

$$
\omega_{g}^{a}\left(\delta, \delta^{\prime}\right)=\delta \theta^{a}\left(\delta^{\prime}\right)-\delta^{\prime} \theta^{a}(\delta) .
$$

The charge is given by integrating

$$
\Omega\left(\delta_{\xi}, \delta\right)=: \delta Q_{\xi} .
$$

\section{LINEARIZED GRAVITY IN SIX DIMENSIONS}

Before considering arbitrary even dimensions, it will be useful to first study the case of six dimensions in detail, which is done in this section. In Sec. III. A, we state the falloffs and the constraints imposed by the Einstein's equations. In Sec. III. B, symplectic potential is evaluated and turns out to be divergent as $t \rightarrow \infty$. We will then consider the problematic (diverging as $t \rightarrow \infty$ ) piece of symplectic potential in Sec. III. C. The charge contribution from this problematic piece will also appear to be divergent. However, we will impose some restrictions on the behavior of free radiative data, $C_{A B}^{(0)}$, near $\mathcal{I}_{ \pm}^{+}$, which will render it finite. Section III. D evaluates the charge contribution coming from the nonproblematic (finite as $t \rightarrow \infty$ ) portion of symplectic potential. In Sec. III. E, adding the charge contributions from the finite and divergent pieces of $\theta^{t}$, we will get the total soft charge. In the linearized gravity under consideration, the hard charge only comes from the matter. It is evaluated and added to soft charge in Sec. III. F to obtain the total asymptotic charge. It matches the charge expected from the leading soft graviton theorem in six dimensions. Section III. G discusses the generalized CK constraints in six dimensions. The calculation is done in the spirit of Ref. [15].

\section{A. Boundary conditions and constraints from Einstein's equations}

In six dimensions, the boundary conditions for asymptotically flat spacetimes are

$g_{u u}=-1+O\left(r^{-1}\right), \quad g_{u r}=-1+O\left(r^{-2}\right)$,

$g_{u A}=O(1), \quad g_{A B}=r^{2} \gamma_{A B}+O(r), \quad R_{u и}=O\left(r^{-4}\right)$,

$R_{u r}=O\left(r^{-5}\right), \quad R_{u A}=O\left(r^{-4}\right), \quad R_{r r}=O\left(r^{-6}\right)$

$R_{r A}=O\left(r^{-5}\right), \quad R_{A B}=O\left(r^{-4}\right)$.

Using linearized Einstein's equations (9) in Bondi coordinates, we get the following constraints:

$$
M^{(1)}=0, \quad M^{(2)}=-\frac{1}{2} D^{A} U_{A}^{(1)} .
$$

The $R_{r A}$ equations give
$U_{A}^{(0)}=-\frac{1}{6} D^{B} C_{B A}^{(-1)}, \quad U_{A}^{(1)}=-\frac{1}{3} D^{B} C_{B A}^{(0)}$,

$U_{A}^{(2)}=-\frac{3}{4} D^{B} C_{B A}^{(1)}$.

The $R_{A B}$ equations lead to

$$
\partial_{u} C_{A B}^{(-1)}=0
$$

$R_{u и}$ equations give

$\frac{1}{2}\left[D^{2}-2\right] M^{2}+\partial_{u} D^{a} U_{a}^{(2)}+2 \partial_{u} M^{(3)}+T_{u u}^{M(4)}=0$,

where $T_{u u}^{M(4)}$ is the $\mathcal{O}\left(r^{-4}\right)$ component of matter stressenergy tensor. $C_{A B}^{(0)}$ is free, unconstrained data.

\section{B. Symplectic potential}

Let us now proceed with the derivation of asymptotic charge by employing the covariant phase space approach (see Sec. II D). The first step is to compute the symplectic potential. We will find that it is divergent as $t \rightarrow \infty$.

We have (as discussed in Sec. II D)

$$
\theta^{t}=\theta^{r}+\theta^{u}
$$

Doing this computation (the details of which are deferred to Appendix A 1), one gets

$\theta^{t}=\frac{1}{2} r^{4} \sqrt{\gamma}\left[\delta U^{A} g_{A B} \partial_{r} U^{B}-\delta g^{A B}\left(D_{(A} U_{B)}+\frac{1}{2} \partial_{u} g_{A B}\right)\right]$.

From the above equation, it is clear that $\theta^{t}$ has divergent terms since, if one expands the metric components in powers of $r$, the terms inside the square brackets are of the order $r^{-3}$, while there is $r^{4}$ sitting outside the square brackets. Thus, there are divergences as $t \rightarrow \infty$ (since $r=t-u$ ). We are now going to compute the charge from $\theta^{t}$. We will see that there are no divergent contributions to the charge, even from the divergent piece of $\theta^{t}$, once suitable falloffs, in $u$, on the free radiative data $\left(C_{A B}^{(0)}\right)$ are imposed.

From now on, sphere indices will be raised and lowered with $\gamma_{A B}$.

\section{Soft charge contribution from divergent part of $\theta^{t}$}

It will be helpful to study the divergent pieces in the symplectic potential separately from the finite pieces and see how the right falloffs near $\mathcal{I}_{ \pm}^{+}$kill the divergences and give some finite contribution to the charge. 
Expanding the metric components in powers of $r$ (Sec. II A), we find that the potentially divergent (with positive powers of $r$ ) part of $\theta^{t}, \theta_{D}^{t}$ (say), is

$$
\begin{aligned}
\theta_{D}^{t}= & \frac{r^{4}}{2} \sqrt{\gamma}\left[\delta U_{A}^{(0)} \partial_{r}\left(\frac{U^{A(0)}}{r^{2}}\right)\right. \\
& \left.+\frac{\delta C^{A B(-1)}}{r^{3}}\left(D_{(A} U_{B)}^{(0)}+\frac{r}{2} \partial_{u} C_{A B}^{(-1)}+\frac{1}{2} \partial_{u} C_{A B}^{(0)}\right)\right] .
\end{aligned}
$$

Now, using Einstein's equations (26) and ignoring total variations, we have

$$
\theta_{D}^{t}=\frac{\sqrt{\gamma}}{2}\left[r \delta C^{A B(-1)}\left(D_{(A} U_{B)}^{(0)}+\frac{1}{2} \partial_{u} C_{A B}^{(0)}\right)\right] .
$$

Substituting for $U_{B}^{(0)}(25)$, we see that the first term is a total variation. Total variations do not contribute to the symplectic form (or to the charge) since the symplectic form involves antisymmetrization of the variations. For the second term, substitute $r=t-u$. Then, up to total variations,

$$
\theta_{D}^{t}=\frac{1}{4} \sqrt{\gamma}(t-u) \partial_{u} C_{A B}^{(0)} \delta C^{A B(-1)} .
$$

Integrating by parts in $u$,

$\theta_{D}^{t}=\frac{1}{4} \sqrt{\gamma} C_{A B}^{(0)} \delta C^{A B(-1)}+\partial_{u}\left(\frac{1}{4} \sqrt{\gamma}(t-u) C_{A B}^{(0)} \delta C^{A B(-1)}\right)$.

Let us call the contribution to the symplectic structure $\Omega^{D}$. Thus, for supertranslations, the form of variations (15) leads to

$$
\begin{aligned}
\Omega^{D}\left(\delta_{\xi}, \delta\right)= & \lim _{t \rightarrow \infty}\left[-\int_{\mathcal{I}^{+}} \frac{\sqrt{\gamma}}{2} \delta C_{A B}^{(0)} D_{A} D_{B} f\right. \\
& \left.-\int_{\mathcal{I}^{+}} \partial_{u}\left((t-u) \frac{\sqrt{\gamma}}{2} \delta C_{A B}^{(0)} D_{A} D_{B} f\right)\right] .
\end{aligned}
$$

In the limit $t \rightarrow \infty$, the above expression is generically divergent. To avoid this, in the limit $t \rightarrow \infty$, we impose the condition

$$
\begin{aligned}
D^{A} D^{B} C_{A B}^{(0)}(u=+\infty, \hat{x}) & =D^{A} D^{B} C_{A B}^{(0)}(u=-\infty, \hat{x}) \\
& =O\left(|u|^{-1-\epsilon}\right),
\end{aligned}
$$

where $\hat{x}$ labels a point on the conformal sphere.

Conditions (35) ensure the vanishing of the potentially divergent total derivative term after $u$ integration. It should be noted that in the linearized regime the falloffs (35) are consistent with the action of supertranslations; i.e., if one starts with a portion of radiative phase space parametrized by the free radiative data $\left(C_{A B}^{(0)}\right)$ of the form given by Eq. (35) (along with nonradiative/"kinematical" data $C_{A B}^{(-1)}$ ), supertranslations preserve it. This can be easily seen in the linearized regime by recalling $\delta_{\xi} C_{A B}^{(0)}=0(15){ }^{3}$

Imposing the above conditions (35) leaves us with a finite contribution to the supertranslations charge. We will denote it with superscript $\mathrm{D}$ to indicate that it is the contribution from the divergent (as $t \rightarrow \infty$ ) part of $\theta^{t}$,

$$
Q_{\xi}^{D}=-\int_{\mathcal{I}^{+}} \frac{\sqrt{\gamma}}{2} D_{A} D_{B} C_{A B}^{(0)} f
$$

\section{Soft charge contribution from the finite part of $\theta^{t}$}

The finite part of $\theta^{t}$ (terms with $r^{0}$ ) is

$$
\begin{aligned}
\theta_{F}^{t}= & \frac{\sqrt{\gamma}}{2}\left[\delta U^{(1)} \cdot U^{(0)}+\left(\delta C^{(-1)} \cdot D U^{(1)}-\delta\left(D U^{(0)}\right) \cdot C^{(0)}\right)\right. \\
& \left.+\frac{1}{2}\left(\partial_{u} C^{(1)} \cdot \delta C^{(-1)}+\partial_{u} C^{(0)} \cdot \delta C^{(0)}\right)\right]
\end{aligned}
$$

after ignoring total variations. Note that the last term does not contribute to the symplectic form for supertranslations since $\delta C_{\xi}^{(0)}=0$. Further simplifying, using (25), terms contributing to charge are

$$
\theta_{t}^{F}=\sqrt{\gamma}\left[\delta C_{D}^{A(-1)}\left(-\frac{1}{18} D^{D} D \cdot C_{A}^{(0)}+\frac{1}{4} \partial_{u} C_{A B}^{(1)}\right)\right] .
$$

Using Einstein equations (in the absence of matter), D.D.C ${ }^{(1)}=0$ (see Ref. [29]), we get

$$
Q_{\xi}^{F}=\int_{\mathcal{I}^{+}} \frac{\sqrt{\gamma} f}{3}\left(\frac{D^{2}}{4}+1\right) D^{A} D^{B} C_{A B}^{(0)} .
$$

\section{E. Total soft charge}

From (36) and (39), the total soft charge for supertranslations is

$Q_{\xi}^{\text {soft }}=Q_{\xi}^{D}+Q_{\xi}^{F}=\int_{\mathcal{I}^{+}} \frac{\sqrt{\gamma} f}{12}\left(D^{2}-2\right) D^{A} D^{B} C_{A B}^{(0)}$.

\footnotetext{
${ }^{3}$ However, under the full action of supertranslations, i.e., including the pieces which are homogeneous in the fields and contribute to the hard part of the charge, the falloffs are violated (more on this is discussed in Sec. V). This is not satisfactory, and one possible resolution may be to add boundary counterterms to the action to cancel the divergences instead of demanding the falloffs (35).
} 


\section{F. Total charge in linearized gravity}

Having found the soft charge, we now turn to the hard charge. When there is coupling to matter, we assume the falloffs of $T_{\mu \nu}$ to be same as those of $R_{\mu \nu}$ as in Ref. [23]. Then, the hard contribution to the charge coming from matter is given by

$$
Q_{\xi}^{\text {hard }}=\lim _{t \rightarrow \infty} \int_{\Sigma_{t}} r^{D-2} \mathrm{~d} u \mathrm{~d} \Omega_{D-2} n_{\Sigma_{t}}^{\mu} T_{\mu \nu}^{M} \xi^{\nu},
$$

which yields, for supertranslations,

$$
Q_{\xi}^{\text {hard }}=\int_{\mathcal{I}^{+}} \sqrt{\gamma} f(z) T_{u u}^{M(4)} .
$$

Therefore, the total charge is

$$
\begin{aligned}
Q_{\xi}= & Q_{\xi}^{\text {soft }}+Q_{\xi}^{\text {hard }}=\int_{\mathcal{I}^{+}} \frac{\sqrt{\gamma} f(z)}{12}\left(D^{2}-2\right) D^{A} D^{B} C_{A B}^{(0)} \\
& +\int_{\mathcal{I}^{+}} \sqrt{\gamma} f(z) T_{u u}^{M(4)} .
\end{aligned}
$$

$Q_{\xi}$ matches with the soft charge obtained in Ref. [23] by starting from the leading soft graviton theorem in six dimensions and writing it as a Ward identity. ${ }^{4}$

This can also be obtained as an "electric charge" using electric components of Weyl tensor as shown in Appendix B.

\section{G. Generalized CK constraints}

In four dimensions, there are $d(d-3) / 2=2$ leading soft graviton theorems, one corresponding to each helicity. But we have only one canonical asymptotic charge corresponding to supertranslations, giving rise to only one soft theorem. The counting is rescued by the presence of one CK constraint [30], which is necessary for a well-defined scattering problem [9]. Similarly, in six dimensions, there are $d(d-3) / 2=9$ leading soft graviton theorems. But we have only one canonical charge, giving rise to only one independent soft theorem. So, analogously to four dimensions, we expect that there should be eight generalized CK constraints in six dimensions. Six out of these were given in Ref. [23] by the requirement of the vanishing of the magnetic component of the Weyl tensor near boundaries of null infinity,

\footnotetext{
${ }^{4}$ While comparing with Ref. [23], one should keep in mind that we are working in the units $8 \pi G=1$. Thus, for us, $\kappa^{2}=$ $32 \pi G=4$.

${ }^{5}$ In four dimensions, the origin of the CK constraints can be understood as the requirement that the magnetic charge (as defined in Ref. [31]) vanishes. However, in higher dimensions, their origin is not so well understood in this way. A straightforward analog of this magnetic charge vanishes identically in higher dimensions giving no constraints (also see Appendix B).
}

$$
C_{u r A B}(u= \pm \infty, \hat{x})=\mathcal{O}\left(r^{-2}\right) .
$$

The $\mathcal{O}\left(r^{-1}\right)$ component of this equation gives

$$
D_{A} U_{B}^{(0)}-D_{B} U_{A}^{(0)}=0 .
$$

It was pointed out in Ref. [23] that this condition together with (26) implies that $C_{A B}^{(-1)}$ is a pure supertranslation. Now, we propose that $u$ falloffs (35) that were imposed to ensure finiteness of charge are a part of the CK constraints in six dimensions. This gives us two more CK type constraints. Together with (45), they make up a total of eight generalized CK constraints needed in six dimensions.

\section{GENERALIZATION TO ARBITRARY EVEN DIMENSIONS}

In this section, we generalize our analysis to arbitrary higher even dimensions. We first give the conditions necessary for a finite, well-defined charge. After that, the strategy is the same as in six dimensions. We use covariant phase space analysis and first analyze the symplectic potential in arbitrary dimensions. It has divergences, but we go ahead and compute the charge. The conditions mentioned in (IV A) then ensure that it is finite and equal to the expected charge from the leading soft graviton theorem.

\section{A. Conditions for finiteness of the charge}

Einstein's equations determine D.D.C $C^{(n)}, 0<n<m-3$, recursively in terms of D.D. $C^{(-1)}$ and determine D.D.C $C^{(n)}$, $m-1<n<2 m-3$, recursively in terms of D.D. $C^{(m-2)}$ (see Ref. [29]),

$$
\begin{aligned}
\partial_{u} D \cdot D \cdot C^{(n)} & =\mathcal{D}_{n, m} \text { D.D.C } C^{(n-1)}, \quad 0 \leq n \leq m-3, \\
m-1 & \leq n \leq 2 m-3,
\end{aligned}
$$

where

$$
\begin{aligned}
\mathcal{D}_{n, m}= & \frac{n(2 m-n-3)}{2(n+2)(-2 m+n+1)(-m+n+2)} \\
& \times\left(D^{2}-(n+1)(2 m-n-2)\right) .
\end{aligned}
$$

As is clear from the above equation, D.D.C $C^{(n)}$ is determined in terms of D.D.C. $C^{(n-1)}$ but up to an "integration constant," i.e., a function on the sphere. Now, we will, basically, demand the vanishing of these integration constants. Furthermore, we will impose two conditions on the falloff behavior of the free radiative data, $C^{(m-2)}$, near the (two) boundaries of $\mathcal{I}^{+}$. More concretely, the conditions are

$$
\begin{aligned}
& \text { D.D.C }=u^{n+1}\left(\prod_{j=0}^{n} \mathcal{D}_{j, m}\right) \text { D.D.C. }{ }^{(-1)}, \\
& 0 \leq n \leq m-3, \\
& \text { D.D. }\left.C^{(m-2)}\right|_{(u= \pm \infty, \hat{x})} \backsim \mathcal{O}\left(|u|^{-m+1-\epsilon)}\right),
\end{aligned}
$$




$$
\begin{aligned}
& \text { D.D. }\left.C^{(m-2+n)}\right|_{(u= \pm \infty, \hat{x})} \backsim \mathcal{O}\left(|u|^{-m+1+n-\epsilon)}\right), \\
& 1 \leq n \leq m-2, \quad \epsilon>0 .
\end{aligned}
$$

These $((m-2)+2+(m-2)=2 m-2) \quad$ conditions, together, will ensure the finiteness of the charge as we shall see now.

\section{B. Symplectic potential}

Calculating symplectic potential in arbitrary dimensions, throwing away unnecessary terms, and using Einstein's equations, the part of $\theta^{t}$ that contributes to the soft charge is (for details see, Appendix A 1)

$$
\begin{aligned}
\theta^{t}= & \frac{r^{2 m} \sqrt{\gamma}}{2} \sum_{n=0}^{n=2 m-3}\left[\frac{\delta C^{A B(-1)} D_{A} D \cdot C_{B}^{(n)}}{r^{n+4}} \mathrm{opt}_{n, m}\right. \\
& \left.+\frac{1}{2} \frac{\delta C^{(-1)}}{r^{n+3}} \cdot \partial_{u} C^{(n)}\right]
\end{aligned}
$$

where

$$
\mathrm{opt}_{k, m}=\frac{(k+1) m}{(k+3)(2 m-1)(k-2 m+2)} .
$$

For most values of $n$ (just by counting the power of $r$ ), generically, there are divergent terms in $\theta^{t}$ as $t \rightarrow \infty$. However, the conditions (48), (49), and (50) ensure that the charge is finite as we will now see.

\section{Soft charge}

The soft charge can be computed as explained above (Sec. II D). It will only have terms containing D.D.C ${ }^{(n)}$ since it has to be a scalar, linear in $C_{A B}^{(n)}$, and because of tracelessness of $C_{A B}^{(n)}$ s. An easy computation then gives

$$
\begin{aligned}
\delta Q_{\xi}^{D} & =\int_{\mathcal{I}^{+}} \frac{\sqrt{\gamma}}{2} \sum_{n=m-2}^{n=2 m-3}\left[\frac{1-2 m}{m} \frac{\left(D^{2}+2 m\right) D \cdot D \cdot \delta C^{(n)}}{r^{-2 m+n+4}} \mathrm{opt}_{n, m}\right. \\
& \left.-\frac{\partial_{u} D \cdot D \cdot \delta C^{(n)}}{r^{-2 m+n+3}}\right] f(z) .
\end{aligned}
$$

Note that the sum starts from $m-2$. This is because of Eq. (48), which gives $C_{A B}^{(0<n<m-2)}$ in terms of $C_{A B}^{(-1)}$, making the terms with $n<m-2$ (in (51) total variations that do not contribute to the charge.

Now, expressing the charge in terms of the free radiative data, $C_{A B}^{(m-2)}$, using Einstein's equations (46) (see Appendix A 2), we have

$$
\begin{aligned}
\delta Q_{\xi}^{D} & =\int_{\mathcal{I}^{+}} \frac{\sqrt{\gamma}}{2} \sum_{n=m-2}^{n=2 m-4}\left[\left(\frac{1-2 m}{m} \frac{\left(D^{2}+2 m\right)}{r^{-2 m+n+4}} \text { opt }_{n, m}\right.\right. \\
& \left.\left.-\frac{2 m-n-3}{r^{-2 m+n+4}}\right) \prod_{i=m-1}^{n}\left(\int d u \mathcal{D}_{i, m}\right) D \cdot D \cdot \delta C^{(m-2)} f(z)\right],
\end{aligned}
$$

where $\mathcal{D}_{i, m}$ is a differential operator on the sphere defined by Eq. (47) and we take $\prod_{i=m-1}^{m-2}\left(\int d u \mathcal{D}_{m-2, m}\right):=1$. The charge appears to be divergent since $2 m-4 \geq n$, but let us use a generalization of the trick used in Sec. III C. To this end, we substitute $r=t-u$ and integrate each term in (54) by parts (multiple times) with respect to $u$ until all the factors of $r$ are removed. For instance,

$$
\begin{aligned}
\int d u \frac{D \cdot D \cdot \delta C^{(m-2)}}{r^{-2 m+n+4}}= & (2 m-n-4) ! \int d u I^{(2 m-n-4)} \\
& \times\left(D \cdot D \cdot \delta C^{(m-2)}\right),
\end{aligned}
$$

where $I^{(2 m-n-4)}=\left(\int d u\right)^{(2 m-n-4)}$ is an integral operator denoting the $(2 m-n-4)$ th antiderivative of the argument with respect to $u$. All the boundary terms vanish by the conditions (49) and (50), which can alternatively be written as the following falloff conditions near $\mathcal{I}_{ \pm}^{+}$:

$$
\begin{aligned}
& \left.I^{(k)}\left(D . D . \delta C^{(m-2)}\right)\right|_{(u= \pm \infty, \hat{x})} \backsim \mathcal{O}\left(|u|^{-m+1+k-\epsilon)}\right), \\
& 0 \leq k \leq m-2, \quad \epsilon>0 .
\end{aligned}
$$

Note that these boundary conditions are preserved by the supertranslations in the linearized regime in even $d>4$ (see footnote 3 and Sec. V) because supertranslations do not act on $C_{A B}^{(n \geq 0)}$ (up to homogeneous terms which lead to nonlinear terms in the charge) (15).

Now, from Eqs. (54) and (55), after simplification (see Appendix A 2), we obtain

$$
\begin{aligned}
Q_{\xi}^{\mathrm{soft}}= & \frac{1}{(2 m-1)} \frac{2^{-m}}{\Gamma(m)} \int_{\mathcal{I}^{+}} \sqrt{\gamma} f(z) \\
& \times \prod_{l=m+1}^{2 m-1}\left(D^{2}-(2 m-l)(l-1)\right) I^{(m-2)}\left(D . D . C^{(m-2)}\right) .
\end{aligned}
$$

The charge is the same as in Ref. [23], as can be seen using the integral property of Fourier transforms as follows. Each antiderivative brings down a power of frequency. ${ }^{6}$ The final $u$ integral over $\mathcal{I}^{+}$picks out the zero mode of the $(m-2)$ th antiderivative of D.D.C $C^{(m-2)}$.

\footnotetext{
${ }^{6}$ The Fourier transform of an integral also involves a boundary term which is basically the zero mode of the unintegrated function times a delta function. This term vanishes due to (56).
} 


\section{Total charge}

As in six dimensions, when we have matter coupled to the theory, we get a hard matter contribution to the charge, which is

$$
Q_{\xi}=\int_{\mathcal{I}^{+}} \sqrt{\gamma} f(z) T_{u u}^{M(2 m)}
$$

The total charge is, thus,

$$
\begin{aligned}
Q_{\xi}= & Q_{\xi}^{\text {soft }}+Q_{\xi}^{\text {hard }}=\frac{1}{(2 m-1)} \frac{2^{-m}}{\Gamma(m)} \int_{\mathcal{I}^{+}} \sqrt{\gamma} f(z) \\
& \times \prod_{l=m+1}^{2 m-1}\left(D^{2}-(2 m-l)(l-1)\right) I^{(m-2)}\left(D . D . C^{(m-2)}\right) \\
& +\int_{\mathcal{I}^{+}} \sqrt{\gamma} f(z) T_{u u}^{M(2 m)}
\end{aligned}
$$

This can also be obtained as an "electric charge" using electric components of the Weyl tensor as shown in Appendix B.

\section{E. Generalized CK constraints}

In $d$ dimensions, there are $d(d-3) / 2$ leading soft graviton theorems, one corresponding to each helicity. But we have only one canonical asymptotic charge corresponding to supertranslations, giving rise to only one independent soft theorem. So, we expect that there should be $d(d-3) / 2-1$ generalized CK constraints in $d$ dimensions. $(d-2)(d-3) / 2$ out of these were given in Ref. [23] by the requirement of vanishing of the magnetic component of Weyl tensor near boundaries of null infinity,

$$
C_{u r A B}(u= \pm \infty, \hat{x})=\mathcal{O}\left(r^{-2}\right) .
$$

The $\mathcal{O}\left(r^{-1}\right)$ component of this equation gives

$$
D_{A} U_{B}^{(0)}-D_{B} U_{A}^{(0)}=0 .
$$

This condition together with (26) implies that $C_{A B}^{(-1)}$ is a pure supertranslation. Now, as in six dimensions, we propose the conditions (48), (49), and (50) (which ensure finiteness of the charge) to be some of the CK constraints in arbitrary dimensions. This gives us $2 m-2=d-4$ more CK type constraints. Together with (61), they make up the required total of $d(d-3) / 2-1(=(d-2)(d-3) / 2+(d-4))$ generalized CK constraints needed in $d$ dimensions.

\section{PROBLEMS IN GOING BEYOND LINEARIZED REGIME}

In this work, we have restricted ourselves only to the linearized gravity coupled to scalar matter. There are several important issues in the full nonlinear theory of which the resolution is beyond the scope of this paper. To understand these issues, let us consider the case of six dimensions for concreteness. In the full theory, under supertranslations, the radiative data transform as

$$
\begin{aligned}
\delta C_{A B}^{(0)}= & -\frac{C_{A B}^{(-1)}}{8} D^{2} f+\gamma_{A B}\left(\frac{2}{3} D^{D} f D \cdot C_{D}^{(-1)}+\frac{D^{A} D^{C} f C_{C D}^{(-1)}}{2}\right) \\
& +\frac{D_{A} f D \cdot C_{B}^{(-1)}}{6}-\frac{D_{A} D^{C} f C_{C B}^{(-1)}}{2}-f \frac{\partial_{u} C_{A B}^{(0)}}{2} .
\end{aligned}
$$

The right-hand side of the above equation contains $u$ independent pieces (all the terms containing $C^{(-1)}$ ). Thus, this transformation violates the $u$ falloff conditions that were imposed to ensure the finiteness of the soft charge (35),

$$
\begin{aligned}
D^{A} D^{B} C_{A B}^{(0)}(u=+\infty, \hat{x}) & =D^{A} D^{B} C_{A B}^{(0)}(u=-\infty, \hat{x}) \\
& =O\left(|u|^{-1-\epsilon}\right) .
\end{aligned}
$$

Instead of imposing these falloffs, it may be possible to settle this issue by adding some counterterms in the action, but this remains to be investigated.

Moreover, it turns out that in full theory generically there are quadratic terms in the charge which are functionals of $C_{A B}^{(0)}$ and $C_{A B}^{(-1)}$. Being linear in free radiative data $C_{A B}^{(0)}$, they would potentially contribute to the soft charge, but as $C^{(-1)}$ parametrize vacuua of the theory, an understanding of such terms would require us to understand coupling of the "kinematic data" $C^{(-1)}$ with radiative data $C^{(0)}$. In the absence of such understanding, our analysis of asymptotic symmetries of full theory which is consistent with the soft graviton theorem remains incomplete.

\section{CONCLUSIONS AND OUTLOOK}

Supertranslation symmetries in higher even dimensions are in an ambivalent state. Detailed analysis in classical gravity literature suggests that an asymptotic symmetry group of general relativity in higher (even) dimensions is precisely the Poincaré group. Primary reasons for this rather stringent constraint on the asymptotic structure of the theory are the following. Unlike in four dimensions, supertranslations are not tied to gravitational radiation (and more precisely the memory effect) in higher even dimensions. ${ }^{7}$ This enables one to choose boundary conditions that permit radiating solutions without allowing supertranslations. Although, in principle, one can relax these boundary conditions, such conditions were thought to lead to an ill-defined asymptotic charge due to a divergent symplectic current.

However, soft graviton theorems, which in four dimensions are statements about (asymptotic) symmetries of the $\mathcal{S}$-matrix, are robust constraints on perturbative quantum gravity in higher dimensions. In fact, their status is far more transparent in higher dimensions due to the absence of infrared divergence and well-defined-ness of the perturbative $\mathcal{S}$-matrix. In Ref. [23], strong evidence was given for the

\footnotetext{
${ }^{7}$ The case of odd dimensions is far more subtle, as in the presence of radiation, there does not exist a notion of null infinity and even formulating questions regarding asymptotic symmetries seems far more difficult [32].
} 
existence of supertranslations in higher even dimensions precisely by writing the leading soft graviton theorem as a Ward identity of the supertranslation symmetry. What has, however, been absent so far in the literature is an analysis of relaxed boundary conditions in classical gravity which admits supertranslations and leads to a well-defined asymptotic charge at $\mathcal{I}$, the Ward identities of which are precisely the one formulated in Ref. [23]. We have taken certain steps toward filling this gap in the paper.

Namely, we obtain the (asymptotic) charge for supertranslations in arbitrary even dimensions using a covariant phase space approach in the linearized gravity coupled to massless matter. We have shown that this charge is well defined and matches the results expected from the leading soft graviton theorem [23]. One may wonder how our analysis (albeit in the context of linearized perturbations) bypasses the rigorous no-go results obtained in the literature. That is, the use of relaxed boundary conditions proposed in Ref. [23] allows for supertranslations. This, however, appears to lead to an ill-defined charge in accordance with the analysis in Ref. [1]. We cured this by:

(i) imposing appropriate falloffs in $\mathrm{u}$ for the free radiative data near the boundaries of $\mathcal{I}^{+}\left(\mathcal{I}_{ \pm}^{+}\right)$(49),

(ii) imposing conditions for the vanishing of integration constants (48), (50) that appear while solving Einstein's equations (46).

We further proposed these conditions to be the generalized CK constraints along with the conditions of vanishing of the magnetic part of the Weyl tensor (61) near $\mathcal{I}_{ \pm}^{+}$. They were shown to match the number of constraints required to establish the uniqueness of the leading soft graviton theorem. One may worry about whether or not the $u$ falloffs (49) allow for interesting physical situations. With regard to this, it can be easily checked that at least the gravity waves radiated from the classical scattering processes (which were studied in Ref. [33]) respect these falloffs. We expect them to be satisfied in other physically relevant scenarios as well.

However, there are some important open issues that remain open and that pertain to the analysis of asymptotic symmetries in full (as opposed to linearized) theory. Once higher order perturbations are allowed, supertranslations do not appear to respect the $u$ falloffs imposed on the radiative data (62). This could possibly be settled by adding boundary counterterms to the action instead of imposing the $u$ falloffs. However, this issue requires further investigation.

Unlike in four dimensions where the origin of the so-called CK constraints is well understood and is tied to the vanishing of the asymptotic charge associated to the magnetic part of Weyl tensor, no such interpretation seems available in higher dimensions. It will also be interesting to see if our analysis can be generalized to include massive fields along the lines of Ref. [34].

It may also be noted that in six dimensions the structure of the potentially problematic (divergent) terms and the $u$ falloffs imposed to cure them is similar those in Ref. [15] in the case of the subleading soft graviton theorem in four dimensions. This hints at some possible connection between subleading soft graviton theorems in lower dimensions and leading soft graviton theorems in higher dimensions as was mentioned in Ref. [35]. The case of superrotations in higher even dimensions is under investigation.

\section{ACKNOWLEDGMENTS}

I am very grateful to Alok Laddha for suggesting this problem; his guidance, insightful discussions, and numerous contributions throughout, including his critique of the final draft, made this project possible. I would also like to thank Arnab Priya Saha for clarifying many basic and subtle points during several illuminating discussions that we had at the early stages of the project, suggesting the very crucial reference [29], and sharing his ongoing work in odd dimensions [32].

\section{APPENDIX A: DETAILS OF CHARGE COMPUTATION IN HIGHER DIMENSIONS}

\section{Symplectic potential}

Details of this section are valid in all even dimensions. We have

$$
\theta^{r}:=\frac{1}{2} \sqrt{g}\left(g^{b c} \delta \Gamma_{b c}^{r}-g^{r b} \delta \Gamma_{c b}^{c}\right)
$$

Using the linearized version of Christoffel symbols in Ref. [36] (with $\beta=0$ in linearized theory), we get

$$
\begin{aligned}
\theta^{r}= & \frac{1}{2} r^{2 m} \sqrt{\gamma}\left[\delta\left(\partial_{r} M\right)-U^{A} \delta\left(g_{A B} \partial_{r} U^{B}\right)\right. \\
& \left.+g^{A B} \delta\left(D_{(A} U_{B)}+\frac{1}{2} \partial_{u} g_{A B}+\frac{M}{2} \partial_{r} g_{A B}\right)\right] .
\end{aligned}
$$

Neglecting terms which are total variations (since they do not contribute to the symplectic structure), we have

$$
\begin{aligned}
\theta^{r}= & \frac{1}{2} r^{2 m} \sqrt{\gamma}\left[\delta U^{A} g_{A B} \partial_{r} U^{B}-\delta g^{A B}\left(D_{(A} U_{B)}+\frac{1}{2} \partial_{u} g_{A B}\right.\right. \\
& \left.\left.+\frac{M}{2} \partial_{r} g_{A B}\right)\right] .
\end{aligned}
$$

Similarly,

$$
\begin{aligned}
& \theta^{u}:=\frac{1}{2} \sqrt{g}\left(g^{b c} \delta \Gamma_{b c}^{u}-g^{u b} \delta \Gamma_{c b}^{c}\right), \\
& \theta^{u}=\frac{1}{4} r^{2 m} \sqrt{\gamma} \delta\left(\partial_{r} g_{A B}\right),
\end{aligned}
$$

\footnotetext{
${ }^{8}$ Although, in Ref. [36], they were written in the context of four dimensions, they are valid in all dimensions.
} 


$$
\begin{aligned}
\Rightarrow \theta^{t}= & \frac{1}{2} r^{2 m} \sqrt{\gamma}\left[\delta U^{A} g_{A B} \partial_{r} U^{B}-\delta g^{A B}\left(D_{(A} U_{B)}+\frac{1}{2} \partial_{u} g_{A B}\right.\right. \\
& \left.\left.+r(M+1) \gamma_{A B}\right)\right] .
\end{aligned}
$$

Here, we have kept only terms that are quadratic in metric perturbations. This is because we are working in the linearized regime. Tracelessness of $\delta g_{A B}$ now implies

$\theta^{t}=\frac{1}{2} r^{2 m} \sqrt{\gamma}\left[\delta U^{A} g_{A B} \partial_{r} U^{B}-\delta g^{A B}\left(D_{(A} U_{B)}+\frac{1}{2} \partial_{u} g_{A B}\right)\right]$.

Now, we use the fact that $\delta_{\xi} C_{A B}^{(n \geq 0)}=0$ to throw away terms which are of the form $\delta C^{(n \geq 0)} \delta C^{(m \geq 0)}$ since they do not contribute to the charge along with the total variations. We thus get the part of $\theta^{t}$ that contributes

$$
\begin{aligned}
\theta^{t}= & \frac{r^{2 m} \sqrt{\gamma}}{2} \sum_{n=0}^{n=2 m-3}\left[\frac{-(n+1)}{r^{n+4}} \delta U^{(0)} \cdot U^{(n+1)}\right. \\
& +\frac{\delta C^{(-1)}}{r^{n+4}} \cdot D U^{(n+1)}+\frac{\delta C^{(n)}}{r^{n+4}} \cdot D U^{(0)} \\
& \left.+\frac{1}{2} \frac{\delta C^{(-1)}}{r^{n+3}} \cdot \partial_{u} C^{(n)}\right] .
\end{aligned}
$$

Now, Einstein's equation for $R_{r A}$ gives

$$
U_{A}^{(n)}=f_{n, m} D \cdot C_{A}^{(n-1)}, \quad 0 \leq n \leq 2 m-2,
$$

where

$$
f_{n, m}=\frac{(n+1)}{(n+2)(n+1-2 m)} .
$$

Substituting in $\theta^{t}$, we have Eq. (51),

$$
\begin{aligned}
\theta^{t}= & \frac{r^{2 m} \sqrt{\gamma}}{2} \sum_{n=0}^{n=2 m-3}\left[\frac{\delta C^{A B(-1)} D_{A} D \cdot C_{B}^{(n)}}{r^{n+4}} \mathrm{opt}_{n, m}\right. \\
& \left.+\frac{1}{2} \frac{\delta C^{(-1)}}{r^{n+3}} \cdot \partial_{u} C^{(n)}\right],
\end{aligned}
$$

where

$$
\begin{aligned}
\mathrm{opt}_{k, m} & =(k+1) f_{k+1, m} f_{0, m}+f_{1+k, m}-f_{0, m} \\
& =\frac{(k+1) m}{(k+3)(2 m-1)(k-2 m+2)} .
\end{aligned}
$$

\section{Soft charge}

Starting from (53), using the Einstein's equations, the remaining D.D.C $C^{(2 m-3>n \geq m-2)}$ can be expressed in terms of D.D.C $C^{(m-2)}$ (see Ref. [29]). In the absence of matter, D.D. $C^{(2 m-3)}=0$. Other relations are as given by

$$
\partial_{u} \text { D.D.C. }{ }^{(n)}=\mathcal{D}_{n, m} \text { D.D.C. }{ }^{(n-1)}, \quad 0 \leq n \leq 2 m-3,
$$

where

$$
\begin{aligned}
& \mathcal{D}_{n, m}= \frac{n(2 m-n-3)}{2(n+2)(-2 m+n+1)(-m+n+2)} \\
& \times\left(D^{2}-(n+1)(2 m-n-2)\right), \\
& \Rightarrow \text { D.D.C. }{ }^{(n)}=\int d u \mathcal{D}_{n, m} D \cdot D \cdot C^{(n-1)}, \\
& \Rightarrow \text { D.D.C } C^{(n)}=\prod_{i=m-1}^{n}\left(\int d u \mathcal{D}_{i, m}\right) \text { D.D.C } C^{(m-2)}, \\
& n \geq m-1 .
\end{aligned}
$$

A few things are clear here. The last term in (53) can be integrated by parts in $u$, and the boundary term, thus obtained, will vanish for the conditions (49) and (50). With that and using the Einstein equations above, we have (54).

Now, from (54) and (55), we obtain

$$
\begin{aligned}
Q_{\xi}^{\text {soft }}= & (2 m-n-4) ! \int_{\mathcal{I}^{+}} \frac{\sqrt{\gamma}}{2} \sum_{n=m-2}^{2 m-4} \\
& \times\left[\left(\frac{1-2 m}{m}\left(D^{2}+2 m\right) \operatorname{opt}_{n, m}-(2 m-n-3)\right)\right. \\
& \left.\times \prod_{i=m-1}^{n}\left(\mathcal{D}_{i, m}\right) I^{(m-2)}\left(D \cdot D \cdot C^{(m-2)}\right) f(z)\right],
\end{aligned}
$$

where opt ${ }_{n, m}$ and $\mathcal{D}_{i, m}$ are given by (52) and (47), respectively. This tedious-looking expression factorizes and simplifies ${ }^{9}$ to $(57)$

$$
\begin{aligned}
Q_{\xi}^{\text {soft }}= & \frac{1}{(2 m-1)} \frac{2^{-m}}{\Gamma(m)} \int_{\mathcal{I}^{+}} \sqrt{\gamma} f(z) \\
& \times \prod_{l=m+1}^{2 m-1}\left(D^{2}-(2 m-l)(l-1)\right) I^{(m-2)}\left(D \cdot D \cdot C^{(m-2)}\right) .
\end{aligned}
$$

\section{APPENDIX B: ELECTRIC CHARGE}

Analogous to Ref. [15], we define electric charges ${ }^{10}$

$$
Q_{\mathcal{I}}[\xi]:=\lim _{t \rightarrow \infty} \int_{\Sigma_{t}} \partial_{a}\left(\mathcal{E}_{b}^{a} \xi^{b}\right)
$$

where

\footnotetext{
${ }^{9}$ One should be able to prove the equivalence of (57) with (A17) by induction. Although we have not been able to do so, verification for any particular $m$ is easily done using a symbolic manipulation software like Mathematica. We will obtain (57) in another way by computing electric charge as in case of six dimensions.

${ }^{10}$ Reference [31] also defines a magnetic charge which gives the $\mathrm{CK}$ constraint in four dimensions. Its generalization in higher even dimensions vanishes identically (at least in the linearized regime).
} 


$$
\mathcal{E}_{b}^{a}:=-\frac{1}{2 m-1} r \sqrt{g} C_{b r}^{a t}
$$

Here, the relevant components of the Weyl tensor are

$$
C_{u u r}^{u}=-\frac{1}{2} \partial_{r}^{2} M
$$

and

$$
C_{u A r}^{u}=\frac{1}{2}\left(\partial_{r}-\frac{1}{r}\right) \partial_{r} U_{A}
$$

Then, the electric charge defined by (B1) gives

$$
Q_{\mathcal{I}}[\xi]=m \int_{\mathcal{I}^{+}} \partial_{u} M^{(2 m-1)} f(z)
$$

where we have used Einstein's equations and (56). Writing it out in terms of the free radiative data using Einstein's equations, we see that the charge obtained is the same as the one above, which was obtained using symplectic phase space formalism,

$$
\begin{aligned}
Q_{\mathcal{I}}[\xi]= & Q_{\xi}=\frac{1}{(2 m-1)} \frac{2^{-m}}{\Gamma(m)} \int_{\mathcal{I}^{+}} \sqrt{\gamma} f(z) \\
& \times \prod_{l=m+1}^{2 m-1}\left(D^{2}-(2 m-l)(l-1)\right) I^{(m-2)}\left(D \cdot D \cdot C^{(m-2)}\right) \\
& +\int_{\mathcal{I}^{+}} \sqrt{\gamma} f(z) T_{u u}^{M(2 m)} .
\end{aligned}
$$

\section{APPENDIX C: COMMUTATORS}

Some useful commutation relations used above are

$$
\begin{aligned}
{\left[D^{B}, D_{N}\right] C_{A B}^{(0)}=} & 2 m C_{A N}^{(0)} \\
{\left[D^{B}, D_{M}\right] D_{N} C_{A B}^{(0)}=} & D_{M} C_{A N}^{(0)}+2 m D_{N} C_{A M}^{(0)}-q_{M N} D \cdot C_{A}^{(0)} \\
{\left[D^{A}, D_{N}\right] D \cdot C_{A}^{(0)}=} & (2 m-1) D \cdot C_{N}^{(0)} \\
{\left[D^{A}, D_{M}\right] D_{N} D \cdot C_{A}^{(0)}=} & D_{M} D \cdot C_{N}^{(0)}-\gamma_{M N} D \cdot D \cdot C^{(0)} \\
& +(2 m-1) D_{N} D \cdot C_{M}^{(0)} .
\end{aligned}
$$

[1] S. Hollands, A. Ishibashi, and R. M. Wald, BMS supertranslations and memory in four and higher dimensions, Classical Quantum Gravity 34, 155005 (2017).

[2] H. Bondi, M. G. J. Van Der Burg, and A. W. K. Metzner, Gravitational waves in general relativity, VII. Waves from axi-symmetric isolated system, Proc. R. Soc. A 269, 21 (1962).

[3] R. K. Sachs, Gravitational waves in general relativity viii. waves in asymptotically flat space-time, Proc. R. Soc. A 270, 103 (1962).

[4] R. Sachs, Asymptotic symmetries in gravitational theory, Phys. Rev. 128, 2851 (1962).

[5] S. Hollands and A. Ishibashi, Asymptotic flatness at null infinity in higher dimensional gravity, arXiv:hep-th/ 0311178.

[6] S. Hollands and A. Ishibashi, Asymptotic flatness and Bondi energy in higher dimensional gravity, J. Math. Phys. (N.Y.) 46, 022503 (2005).

[7] K. Tanabe, S. Kinoshita, and T. Shiromizu, Asymptotic flatness at null infinity in arbitrary dimensions, Phys. Rev. D 84, 044055 (2011).

[8] A. Strominger, Asymptotic symmetries of Yang-Mills theory, J. High Energy Phys. 07 (2014) 151.

[9] A. Strominger, On BMS invariance of gravitational scattering, J. High Energy Phys. 07 (2014) 152.

[10] T. He, P. Mitra, A. P. Porfyriadis, and A. Strominger, New symmetries of massless QED, J. High Energy Phys. 10 (2014) 112.
[11] D. Kapec, V. Lysov, and A. Strominger, Asymptotic symmetries of massless QED in even dimensions, Adv. Theor. Math. Phys. 21, 1747 (2017).

[12] V. Lysov, S. Pasterski, and A. Strominger, Low's subleading soft theorem as a symmetry of QED, Phys. Rev. Lett. 113, 111601 (2014).

[13] T. He, V. Lysov, P. Mitra, and A. Strominger, BMS supertranslations and Weinberg's soft graviton theorem, J. High Energy Phys. 05 (2015) 151.

[14] D. Kapec, V. Lysov, S. Pasterski, and A. Strominger, Semiclassical Virasoro symmetry of the quantum gravity $\mathcal{S}$-matrix, J. High Energy Phys. 08 (2014) 058.

[15] M. Campiglia and A. Laddha, New symmetries for the gravitational S-matrix, J. High Energy Phys. 04 (2015) 076.

[16] M. Campiglia and A. Laddha, Asymptotic symmetries and subleading soft graviton theorem, Phys. Rev. D 90, 124028 (2014).

[17] M. Campiglia and A. Laddha, Asymptotic symmetries of QED and Weinberg's soft photon theorem, J. High Energy Phys. 07 (2015) 115.

[18] A. Strominger, Lectures on the infrared structure of gravity and gauge theory, arXiv:1703.05448.

[19] S. Weinberg, Infrared photons and gravitons, Phys. Rev. 140, B516 (1965).

[20] A. Sen, Soft theorems in superstring theory, J. High Energy Phys. 06 (2017) 113.

[21] A. Sen, Subleading soft graviton theorem for loop amplitudes, J. High Energy Phys. 11 (2017) 123. 
[22] A. Laddha and A. Sen, Sub-subleading soft graviton theorem in generic theories of quantum gravity, J. High Energy Phys. 10 (2017) 065.

[23] D. Kapec, V. Lysov, S. Pasterski, and A. Strominger, Higher-dimensional supertranslations and Weinberg's soft graviton theorem, Ann. Math. Sci. Appl. 2, 69 (2017).

[24] S. Hollands and R. M. Wald, Conformal null infinity does not exist for radiating solutions in odd spacetime dimensions, Classical Quantum Gravity 21, 5139 (2004).

[25] A. Ashtekar, L. Bombelli, and O. Reula, The covariant phase space of asymptotically flat gravitational fields, in Mechanics, Analysis and Geometry: 200 Years After Lagrange (Elsevier, New York, 1991), pp. 417-450.

[26] J. Lee and R. M. Wald, Local symmetries and constraints, J. Math. Phys. (N.Y.) 31, 725 (1990).

[27] R. M. Wald and A. Zoupas, A general definition of 'conserved quantities' in general relativity and other theories of gravity, Phys. Rev. D 61, 084027 (2000).

[28] G. Compère and A. Fiorucci, Advanced lectures in general relativity, Ph.D. thesis, 2018, https://inspirehep.net/record/ 1649253/files/arXiv:1801.07064.pdf.
[29] A. Campoleoni, D. Francia, and C. Heissenberg, Asymptotic charges at null infinity in any dimension, Universe $\mathbf{4}$, 47 (2018).

[30] D. Christodoulou and S. Klainerman, The Global Nonlinear Stability of the Minkowski Space (Princeton University Press, Princeton, 1993).

[31] M. Campiglia and A. Laddha, Sub-subleading soft gravitons: New symmetries of quantum gravity?, Phys. Lett. B 764, 218 (2017).

[32] A. P. Saha, notes on asymptotic symmetries in odd dimensions (unpublished).

[33] A. Laddha and A. Sen, Gravity waves from soft theorem in general dimensions, J. High Energy Phys. 09 (2018) 105.

[34] M. Campiglia and A. Laddha, Asymptotic symmetries of gravity and soft theorems for massive particles, J. High Energy Phys. 12 (2015) 094.

[35] M. Pate, A.-M. Raclariu, and A. Strominger, Gravitational memory in higher dimensions, J. High Energy Phys. 06 (2018) 138.

[36] G. Barnich and C. Troessaert, Aspects of the BMS/CFT correspondence, J. High Energy Phys. 05 (2010) 062. 